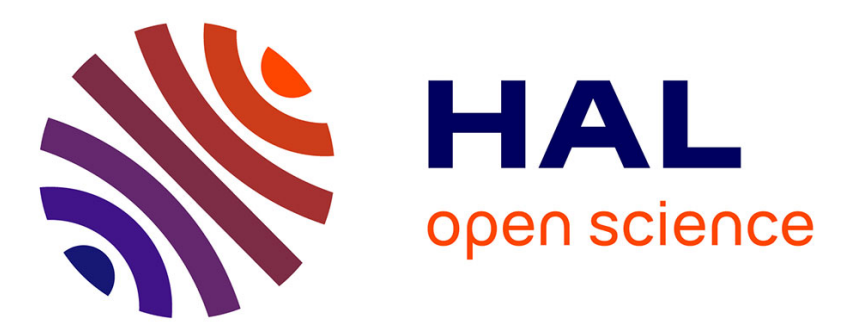

\title{
Bifurcations and stability of amplitude modulated planar oscillations of an orbiting string with internal resonances
}

\author{
Angelo Luongo, Fabrizio Vestroni
}

\section{To cite this version:}

Angelo Luongo, Fabrizio Vestroni. Bifurcations and stability of amplitude modulated planar oscillations of an orbiting string with internal resonances. Nonlinear Dynamics, 1996, 9 (3), pp.305-325. hal-00800614

\section{HAL Id: hal-00800614 https://hal.science/hal-00800614}

Submitted on 14 Mar 2013

HAL is a multi-disciplinary open access archive for the deposit and dissemination of scientific research documents, whether they are published or not. The documents may come from teaching and research institutions in France or abroad, or from public or private research centers.
L'archive ouverte pluridisciplinaire HAL, est destinée au dépôt et à la diffusion de documents scientifiques de niveau recherche, publiés ou non, émanant des établissements d'enseignement et de recherche français ou étrangers, des laboratoires publics ou privés. 


\title{
Bifurcations and Stability of Amplitude Modulated Planar Oscillations of an Orbiting String with Internal Resonances
}

\author{
ANGELO LUONGO and FABRIZIO VESTRONI \\ Dipartimento di Ingegneria delle Strutture, Acque e Terreno, Università dell'Aquila, Monteluco di Roio, \\ 67040 L'Aquila, Italy
}

\begin{abstract}
Nonlinear free transversal oscillations of an orbiting string satellite system are analyzed. They are governed by two partial integro-differential equations with quadratic nonlinearities. The system is weakly nonlinear but in practice works in conditions of nearly simultaneous internal resonance. The ability of truncated models to capture specific phenomena is discussed. By limiting the investigation to the planar motion with a one prevailing component perturbed out-of-plane, two different models with three modes in primary and secondary resonance are adopted. For increasing levels of the system energy, fundamental and bifurcated paths of fixed points are obtained and their stability is investigated. Moreover, periodically amplitude modulated planar motions and their stability for out-of-plane disturbances are studied.
\end{abstract}

Key words: Strings, nonlinear oscillations, multiple modal interactions, motion stability.

\section{Introduction}

The idea of using a tethered satellite deployed from an orbiting Shuttle to develop experiments in space, proposed in the 1960's, has now become a real project thanks to recent technological developments. This explains the large number of papers devoted to the subject that have appeared in dedicated meetings and technical journals [1-7]. Most of the papers concerning this system in the elongated configuration are devoted mainly to linear behaviour. However, physical systems are generally nonlinear and nonlinearities frequently produce a number of unexpected phenomena, particularly in continuous systems [8-10].

The nonlinear transversal oscillations of stretched strings with restrained ends has been the subject of a large number of papers. The nature of the nonlinearities of the hardening type, on account of the stretching, and the sequence of the natural frequencies are such that the internal resonance phenomena are in practice restricted to the single case $\omega_{v_{r}}=\omega_{w_{r}}$ which involves the $r$-th modes in two orthogonal planes [8,11-13]. This makes it possible to use a discrete model based on the only two modes involved in the resonant interaction phenomena.

A large number of continuous dynamical systems have been studied by two-mode models. More than two modes have been considered in few earlier works [14-17] but mainly in recent studies. An exhaustive list of those papers is presented and discussed in [18]. This enlargement of the variable space is fundamental in analyzing the phenomena of multiple resonances. A general discussion of the steady solutions of Hamiltonian three degree-of-freedom systems in conditions of perfect multiple internal resonance is reported in [19].

In the studies of nonlinear oscillations of cables, the interaction of a symmetric in-plane mode with two out-of-plane modes in conditions of simultaneous internal resonances are 


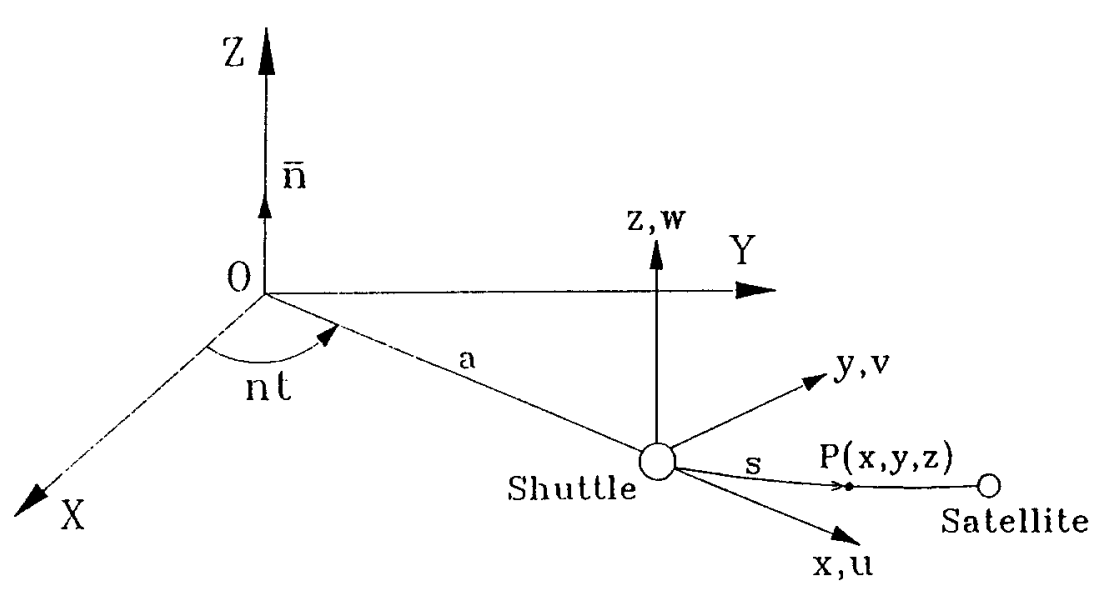

Fig. 1. Tethered satellite system and reference frames.

studied with a three degree-of-freedom model [20]. A four degree-of-freedom model has been proposed for the study of motion involving also the anti-symmetric in-plane mode [21].

The tethered satellite system has a frequency spectrum similar to that of a taut string, apart from the first in-plane and out-of-plane pendulum type modes [6]. On account of the different boundary conditions, cubic terms practically vanish while quadratic terms originating from gyroscopic forces are present; in that it is similar to a cable. Although the nonlinearities are small in comparison with cables or taut strings, the presence of quadratic nonlinearities and the sequence of natural frequencies produce conditions of multiple internal resonances, which make this problem attractive to study, beyond its technical interest.

The present paper examines the transversal nonlinear free oscillations of the undamped tethered satellite system; with respect to a previous work [22], attention is focused on nonstationary motions in conditions of simultaneous internal resonances. The use of discrete models with few degrees-of-freedom sufficiently accurate to describe a particular interaction phenomenon is discussed. Two three degree-of-freedom models are used to study the in-plane motion and its stability for an out-of-plane disturbance, in conditions of simultaneous internal resonances of the type $\omega_{j} \cong 2 \omega_{k}$ and either $\omega_{j} \cong 2 \omega_{l}$ or $\omega_{j} \cong \omega_{l}$.

\section{The Equations of Motion}

The system under study is a string connected at one end to the shuttle and carrying a satellite at the other end. The system orbits in the $X Y$ plane (Figure 1) in equilibrium under centrifugal and gravitational forces. Since the mass of the shuttle is much greater than those of the string and the satellite, the centroid of the system practically coincides with the shuttle; it runs at constant angular velocity $n$ along an orbit assumed circular of radius $a$ related to $n$ through the gravitational constant $\mu_{e}$ by $n^{2}=\mu_{e} / a^{3}$.

An orbiting frame $S x y z$, connected to the shuttle, is introduced as shown in Figure 1. A straight reference configuration $x_{0}(s)$ is assumed where $s$ is the abscissa along the string. The motion is described in this frame by the displacement components $u(s, t), v(s, t), w(s, t)$ measured with respect to the configuration $x_{0}(s)$. The equations of motion were obtained in [23] through Hamilton's principle by using the elongation as a strain measure. 
Let $E A$ be the axial stiffness of the string, $l$ the length, $\mu$ the mass density, $m$ the mass of the satellite. After introducing the dimensionless parameters

$$
\alpha^{2}=\frac{\mu n^{2} l^{2}}{E A}, \quad \gamma=\frac{\mu l}{m}, \quad \xi=\frac{l}{a}
$$

the dimensionless variables

$$
\tilde{x}_{0}=x_{0} / l, \quad \tilde{s}=s / l, \quad \tilde{t}=n t, \quad \tilde{u}=u / l, \quad \tilde{v}=v / l, \quad \tilde{w}=w / l
$$

and the dimensionless static tension $f(s)=\left(\tilde{x}_{0}^{\prime}-1\right) / \alpha^{2}$, the governing equations at the third order, dropping the tilde, read

$$
\begin{aligned}
& \alpha^{2} \ddot{u}-2 \alpha^{2} \dot{v}-3 \alpha^{2} u\left(1-2 \xi x_{0}\right)-\left[u^{\prime}+\frac{v^{2}+w^{\prime 2}}{2}-\left(u^{\prime 2}-\frac{v^{\prime 2}+w^{\prime 2}}{2}\right) u^{\prime}\right]^{\prime}=0 \\
& \alpha^{2} \ddot{v}+2 \alpha^{2} \dot{u}-\alpha^{2}\left[f(s) v^{\prime}\right]^{\prime}-3 \alpha^{2} \xi x_{0} v-\left[\left(u^{\prime}+\frac{v^{\prime 2}+w^{2}}{2}\right) v^{\prime}-u^{\prime 2} v^{\prime}\right]^{\prime}=0 \\
& \alpha^{2} \ddot{w}+\alpha^{2} w\left(1-3 \xi x_{0}\right)-\alpha^{2}\left[f(s) w^{\prime}\right]^{\prime}-\left[\left(u^{\prime}+\frac{v^{\prime 2}+w^{\prime 2}}{2}\right) w^{\prime}-u^{\prime 2} w^{\prime}\right]^{\prime}=0
\end{aligned}
$$

with the boundary conditions:

$$
\begin{aligned}
& u(0, t)=v(0, t)=w(0, t)=0 \\
& \left\{\alpha^{2} \ddot{u}-2 \alpha^{2} \dot{v}-3 \alpha^{2} u\left(1-2 \xi x_{0}\right)+\gamma\left[u^{\prime}+\frac{v^{\prime 2}+w^{\prime 2}}{2}-\left(u^{\prime 2}-\frac{v^{\prime 2}+w^{\prime 2}}{2}\right) u^{\prime}\right]\right\}_{s=1}=0 \\
& \left\{\alpha^{2} \ddot{v}+2 \alpha^{2} \dot{u}+\gamma \alpha^{2} f(s) v^{\prime}-3 \alpha^{2} \xi x_{0} v+\gamma\left[\left(u^{\prime}+\frac{v^{\prime 2}+w^{\prime 2}}{2}\right) v^{\prime}-u^{\prime 2} v^{\prime}\right]\right\}_{s=1}=0 \\
& \left\{\alpha^{2} \ddot{w}+\gamma \alpha^{2} f(s) w^{\prime}+\alpha^{2} w\left(1-3 \xi x_{0}\right)+\gamma\left[\left(u^{\prime}+\frac{v^{\prime 2}+w^{\prime 2}}{2}\right) w^{\prime}-u^{\prime 2} w^{\prime}\right]\right\}_{s=1}=0 .
\end{aligned}
$$

In the previous equations the prime denotes differentiation with respect to $s$, the dot with respect to $t$. The physical meaning of the different terms is easily recognizable: inertial, gyroscopic, elastic and a combination of centrifugal and gravitational forces.

In the study of transversal oscillations a consistent ordering of the terms in equation $\left(3_{1}\right)$, allows omission of inertial and centrifugal-gravitational forces while obtaining an integrodifferential relation among $u, v, w$. By using this relation to eliminate $u(s, t)$, the transversal oscillations of the string are governed by the two equations [22]:

$$
\begin{aligned}
& \ddot{v}-\left[f(s) v^{\prime}\right]^{\prime}-3 \xi x_{0} v-\int_{0}^{s}\left(v^{2}+w^{2}\right)^{\bullet} \mathrm{d} s-\left[\frac{2}{\gamma} \dot{v}_{S} v^{\prime}-v^{\prime} \int_{1}^{s} 2 \dot{v} \mathrm{~d} s\right]^{\prime}=0 \\
& \ddot{w}-\left[f(s) w^{\prime}\right]^{\prime}+\left(1-3 \xi x_{0}\right) w-\left[\frac{2}{\gamma} \dot{v}_{S} w^{\prime}-w^{\prime} \int_{1}^{s} 2 \dot{v} \mathrm{~d} s\right]^{\prime}=0
\end{aligned}
$$


with the boundary conditions:

$$
\begin{aligned}
& v(0, t)=w(0, t)=0 \\
& \ddot{v}_{S}+\gamma f(1) v_{S}^{\prime}-3 \xi x_{0}(1) v_{S}-\int_{0}^{1}\left(v^{2}+w^{\prime 2}\right)^{\bullet} \mathrm{d} s+2 \dot{v}_{S} v_{S}^{\prime}=0 \\
& \ddot{w}_{S}+\gamma f(1) w_{S}^{\prime}+w_{S}\left(1-3 \xi x_{0}(1)\right)+2 \dot{v}_{S} w_{S}^{\prime}=0,
\end{aligned}
$$

where $v_{S}=v(1, t)$ and $w_{S}=w(1, t)$ are the satellite displacements. In view of further developments (5) and (6) are re-written in the more compact form:

$$
\begin{aligned}
& \left\{M(\ddot{v})+L_{v}(v)+B(v, \dot{v})+C(w, \dot{w})\right\} \cdot \delta v=0 \\
& \left\{M(\ddot{w})+L_{w}(w)+D(w, \dot{v})\right\} \cdot \delta w=0,
\end{aligned}
$$

where $M$ and $L$ are linear differential operators of $s$ and $B, C$ and $D$ are bi-linear integrodifferential operators, which are defined in Appendix A.

Before studying the nonlinear equations (7), it is worthwhile to summarize the linear dynamic characteristics of the system. These are obtained from the linearized equations (7) by assuming a suitable mean value $f_{0}$ for the weakly nonlinearly varying tension $f(s)$, a parabolic approximation of which is given in [6]. Equal in-plane and out-of-plane eigenfunctions $\varphi_{k}(s) \equiv \psi_{k}(s) \cong \sin p_{k} s, k=0,1, \ldots$ are found. For $k=0$, in the technical range of the mechanical parameters, $p_{0}$ is less than $\pi / 4$, resulting in a pendulum type mode; for $k>0$, $p_{k} \cong k \pi$. The in-plane and out-of-plane linear frequencies are given by $\omega_{v_{k}}^{2}=p_{k}^{2} f_{0}$ and $\omega_{w_{k}}^{2}=p_{k}^{2} f_{0}+1$, respectively. Apart from the two first pendulum modes, the frequencies of flexible modes practically coincide with those of the taut string; they follow the law $\omega_{k} \simeq k \omega_{1}$ and are the same in the two planes.

In Appendix B an illustrative example is considered and the relevant parameter values are given. Table 1 shows the sequence of the first frequencies of the system for two string lengths which confirm previous observations; in particular the frequencies $\omega_{0}$ of the almost rigid modes are far from the spectrum of the flexible modes.

\section{Models for Modal Interaction Analysis}

The occurrence of multiple modal interaction depends on the sequence of the linear frequencies $\omega_{n}$ and the kinds of nonlinearities. As is well known [10] when quadratic nonlinearities are present the following internal resonance conditions can occur: $\omega_{n} \cong 2 \omega_{m}, \omega_{n} \cong \omega_{m} \pm \omega_{k}$ (primary resonances), and $\omega_{n} \cong \omega_{m}, \omega_{n} \cong 2 \omega_{m} \pm \omega_{k}, \omega_{n} \cong \omega_{k} \pm \omega_{l} \pm \omega_{m}$ (secondary resonances).

For the continuous system under study all these conditions are verified. In the solution reference is made to an approximate discrete model with a limited number of eigenfunctions. Thus, it is important to discuss how to select the modes for the study of particular cases. However this discussion goes beyond the particular free undamped problem dealt with here, because it would concern also the wider case of the damped system under resonant harmonic excitation.

In a general motion a potentially high number of modes are involved, according to the internal resonance phenomenon. However, when a motion with a prevailing modal component has to be dealt with, it is realistic to study the interaction phenomena with a few modes. For 
example, the planar motion is adequately represented by two modal components, the prevailing one of frequency $\omega_{k}$ and the companion mode of frequency $\omega_{j} \cong 2 \omega_{k}$ which is forced by the quadratic term $B(v, \dot{v})$ in $\left(7_{1}\right)$. It is considered acceptable to neglect the contribution of higher modes which are involved by a higher order coupling. Analogous considerations apply for an out-of-plane motion in which the prevailing component is an out-of-plane mode of frequency $\omega_{k}$ and the companion is the in-plane mode of frequency $\omega_{j} \cong 2 \omega_{k}$ forced by the quadratic term $C(w, \dot{w})$ in $\left(7_{2}\right)$, giving rise to a non-planar motion.

In order to analyze the stability of these motions it is necessary to select modes to describe the perturbations. To this end the variational equations of motion are considered:

$$
\begin{aligned}
& \left\{M(\delta \ddot{v})+L_{v}(\delta v)+B(v, \delta \dot{v})+B(\delta v, \dot{v})+C(w, \delta \dot{w})+C(\delta w, \dot{w})\right\} \cdot \delta v=0 \\
& \left\{M(\delta \ddot{w})+L_{w}(\delta w)+D(w, \delta \dot{v})+D(\delta w, \dot{v})\right\} \cdot \delta w=0 .
\end{aligned}
$$

By assuming the disturbance can be described by only one mode in the plane and one out-ofplane, these modes must be chosen so that nonlinear terms are resonant in (8).

For an in-plane motion, $w(s, t) \equiv 0$ and (8) are uncoupled; therefore the stability for an in-plane or out-of-plane disturbance can be studied separately. The frequency $\omega_{i}$ of a planar disturbance $\delta v$ must meet the conditions $\omega_{i} \cong \omega_{k}-\omega_{i}$, i.e. $2 \omega_{i} \cong \omega_{k}$ (primary resonance) or $\omega_{i} \cong \omega_{j}-\omega_{i} \cong 2 \omega_{k}-\omega_{i}$, i.e., $\omega_{i} \cong \omega_{k}$ (secondary resonance). Only the first case is of interest, while the second is trivial since it is known that a two-mode solution cannot be unstable for a disturbance of the same components $[8,21]$, as will be seen later. The frequency $\omega_{l}$ of an out-of-plane disturbance must satisfy similar conditions: $2 \omega_{l} \cong \omega_{k}$ and $\omega_{l} \cong \omega_{k}$, both of interest. In particular, secondary resonance is important when stability of the first flexible in-plane mode $(k=1)$ has to be studied; the primary resonance condition $\omega_{l} \cong \omega_{k} / 2$ cannot occur in this case, as it appears for a sample system from Table 1 in Appendix B.

For spatial motion equations (8) remain coupled and two disturbances $\delta v, \delta w$ must be considered simultaneously. The analysis of resonant terms in the variational equations gives a set of internal resonance conditions. By disregarding trivial cases, the following holds: $\omega_{i} \cong \omega_{k}$, and $\omega_{l} \cong 2 \omega_{k}$.

Summarizing the above considerations, a truncated three-mode model can be satisfactorily referred to in the study of planar motion and its stability, albeit in conditions of simultaneous internal resonances, while a four-mode model has to be adopted for non-planar motion.

In the following two models with three modes are developed to study planar motion under an out-of-plane perturbation in primary and secondary resonance. The amplitude equations are used to analyze both the linearized stability and the nonlinear evolution of motion in the region of unstable solutions. They belong to the class of equations which govern the motion of three degree-of-freedom Hamiltonian systems in 2:4:1 and 1:2:1 internal resonance conditions analyzed in [19]. Spatial motions will be investigated in a later paper.

\section{Amplitude Modulation Equations}

The modulation of the amplitudes of the resonant modes are obtained by the multiple time scale method [24]. The new time scales $t_{n}=\varepsilon^{n} t(n=0,1, \ldots)$ are introduced where $\varepsilon$ is the perturbation parameter; the displacement variable are expanded in two-term series of $\varepsilon$ :

$$
\begin{aligned}
v(s, t) & =\varepsilon v_{0}\left(s, t_{0}, t_{1}\right)+\varepsilon^{2} v_{1}\left(s, t_{0}, t_{1}\right) \\
w(s, t) & =\varepsilon w_{0}\left(s, t_{0}, t_{1}\right)+\varepsilon^{2} w_{1}\left(s, t_{0}, t_{1}\right) .
\end{aligned}
$$


Substituting the previous (9) into the motion equations leads to the following perturbation equations:

$$
\begin{aligned}
& \left\{M\left(d_{00}^{2} v_{0}\right)+L_{v}\left(v_{0}\right)\right\} \cdot \delta v=0 \\
& \left\{M\left(d_{00}^{2} w_{0}\right)+L_{w}\left(w_{0}\right)\right\} \cdot \delta w=0 \\
& \left\{M\left(d_{00}^{2} v_{1}\right)+L_{v}\left(v_{1}\right)\right\} \cdot \delta v=-\left\{B\left(v_{0}, d_{0} v_{0}\right)+C\left(w_{0}, d_{0} w_{0}\right)+2 M\left(d_{01}^{2} v_{0}\right)\right\} \cdot \delta v \\
& \left\{M\left(d_{00}^{2} w_{1}\right)+L_{w}\left(w_{1}\right)\right\} \cdot \delta w=-\left\{D\left(w_{0}, d_{0} v_{0}\right)+2 M\left(d_{01}^{2} w_{0}\right)\right\} \cdot \delta w
\end{aligned}
$$

where $d_{n}=\partial / \partial t_{n}$ and $d_{m n}^{2}=\partial^{2} / \partial t_{m} \partial t_{n}$.

For both three-mode models previously discussed the generating solution is described by three components:

$$
\begin{aligned}
v_{0}(s, t) & =A_{k}\left(t_{1}\right) \varphi_{k}(s) e^{i \omega_{k} t_{0}}+A_{j}\left(t_{1}\right) \varphi_{j}(s) e^{i \omega_{j} t_{0}}+c . c . \\
w_{0}(s, t) & =A_{l}\left(t_{1}\right) \psi_{l}(s) e^{i \omega_{l} t_{0}}+c . c .
\end{aligned}
$$

where c.c. stands for complex conjugate.

Equations (12) are substituted into (11) where $\delta v=\varphi_{k}, \delta v=\varphi_{j}$ and $\delta w=\psi_{l}$ are taken successively; by zeroing secular terms three ODEs in the amplitude $A_{k}, A_{j}$ and $A_{l}$ are obtained.

The two cases of primary and secondary internal resonance between an out-of-plane disturbance and an in-plane motion are dealt with separately; in both cases the two in-plane components are in primary resonance condition which states

$$
2 \omega_{k}=\omega_{j}+\varepsilon \sigma_{k}
$$

where $\sigma_{k}=0(1)$ is a detuning parameter.

\subsection{OUt-of-Plane Mode In PRIMARy ResonanCE Condition}

Primary resonance condition implies that

$$
2 \omega_{l}=\omega_{k}+\varepsilon \sigma_{l}
$$

Elimination of secular terms in (11) gives

$$
\begin{aligned}
& A_{k}^{\prime}-2 b_{k} \bar{A}_{k} A_{j} e^{-i \sigma_{k} t_{1}}-2 c A_{l}^{2} e^{i \sigma_{l} t_{1}}=0 \\
& A_{j}^{\prime}+2 b_{j} A_{k}^{2} e^{i \sigma_{k} t_{1}}=0 \\
& A_{l}^{\prime}-2 d \bar{A}_{l} A_{k} e^{-i \sigma_{l} t_{1}}=0,
\end{aligned}
$$

where overbar denotes complex conjugate, prime $t_{1}$-differentiation and the coefficients are defined in Appendix C.

Introducing the polar form

$$
A_{n}=\frac{1}{2} a_{n}\left(t_{1}\right) e^{i \theta_{n}\left(t_{1}\right)} \quad(n=k, j, l)
$$

and separating in (15) real and imaginary parts, six equations in the three real amplitudes $a_{n}$ and three phases $\theta_{n}$ are obtained. 
The use of the new variables

$$
\begin{aligned}
\gamma_{k} & =2 \theta_{k}-\theta_{j}+\sigma_{k} t_{1} \\
\gamma_{l} & =2 \theta_{l}-\theta_{k}+\sigma_{l} t_{1}
\end{aligned}
$$

makes it possible to reduce the previous system to an autonomous system of five equations in the state variables $z=\left\{a_{k}, a_{j}, a_{l}, \gamma_{k}, \gamma_{l}\right\}^{T}$. They read:

$$
\begin{aligned}
a_{k}^{\prime} & =b_{k} a_{k} a_{j} \cos \gamma_{k}+c a_{l}^{2} \cos \gamma_{l} \\
a_{j}^{\prime} & =-b_{j} a_{k}^{2} \cos \gamma_{k} \\
a_{l}^{\prime} & =-d a_{k} a_{l} \cos \gamma_{l} \\
a_{k} a_{j} \gamma_{k}^{\prime} & =\left(b_{j} a_{k}^{3}-2 b_{k} a_{k} a_{j}^{2}\right) \sin \gamma_{k}+2 c a_{j} a_{l}^{2} \sin \gamma_{l}+\sigma_{k} a_{k} a_{j} \\
a_{l} a_{k} \gamma_{l}^{\prime} & =\left(2 d a_{l} a_{k}^{2}-c a_{l}^{3}\right) \sin \gamma_{l}+b_{k} a_{l} a_{k} a_{j} \sin \gamma_{k}+\sigma_{l} a_{l} a_{k} .
\end{aligned}
$$

A suitable combination of the first three equations furnishes a first integral of motion which states the conservation of energy:

$$
a_{k}^{2}+\frac{b_{k}}{b_{j}} a_{j}^{2}+\frac{c}{d} a_{l}^{2}=I_{k} .
$$

This implies that the trajectories lie on a sphere in the space of the normalized amplitudes $\tilde{a}_{k}=a_{k}, \tilde{a}_{j}=\left(b_{k} / b_{j}\right)^{1 / 2} a_{j}, \tilde{a}_{l}=(c / d)^{1 / 2} a_{l}$.

\subsection{Out-of-Plane Mode in SeCONDARy Resonance Condition}

Secondary resonance conditions implies that

$$
2 \omega_{l}=\omega_{j}+\varepsilon \sigma_{l} .
$$

Elimination of secular terms in (11) gives now

$$
\begin{aligned}
& A_{k}^{\prime}-2 b_{k} \bar{A}_{k} A_{j} e^{-i \sigma_{k} t_{1}}=0 \\
& A_{j}^{\prime}+2 b_{j} A_{k}^{2} e^{i \sigma_{k} t_{l}}-2 c A_{l}^{2} e^{i \sigma_{l} t_{1}}=0 \\
& A_{l}^{\prime}+2 d_{k} \bar{A}_{l} A_{j} e^{-i \sigma_{l} t_{1}}=0,
\end{aligned}
$$

where the coefficients are given in Appendix C.

Introducing the polar form (16) and redefining $\gamma_{l}$ as

$$
\gamma_{l}=2 \theta_{l}-\theta_{j}+\sigma_{l} t_{1}
$$

the following five state equations are obtained:

$$
\begin{aligned}
a_{k}^{\prime} & =b_{k} a_{k} a_{j} \cos \gamma_{k} \\
a_{j}^{\prime} & =-b_{j} a_{k}^{2} \cos \gamma_{k}+c a_{l}^{2} \cos \gamma_{l} \\
a_{l}^{\prime} & =-d a_{l} a_{j} \cos \gamma_{l} \\
a_{k} a_{j} \gamma_{k}^{\prime} & =\left(b_{j} a_{k}^{3}-2 b_{k} a_{k} a_{j}^{2}\right) \sin \gamma_{k}-c a_{k} a_{l}^{2} \sin \gamma_{l}+\sigma_{k} a_{k} a_{j} \\
a_{l} a_{j} \gamma_{l}^{\prime} & =\left(2 d a_{l} a_{j}^{2}-c a_{l}^{3}\right) \sin \gamma_{l}+b_{j} a_{l} a_{k}^{2} \sin \gamma_{k}+\sigma_{l} a_{l} a_{j},
\end{aligned}
$$




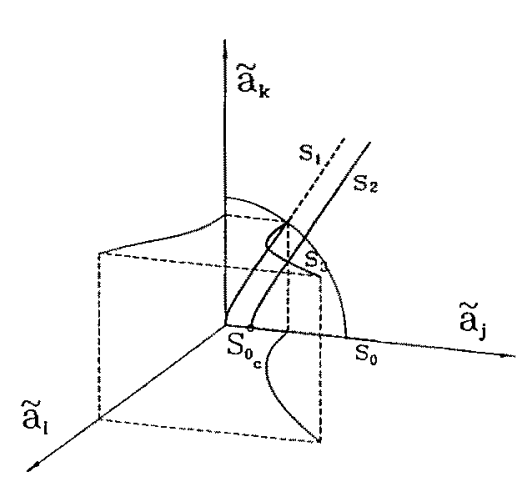

(a)

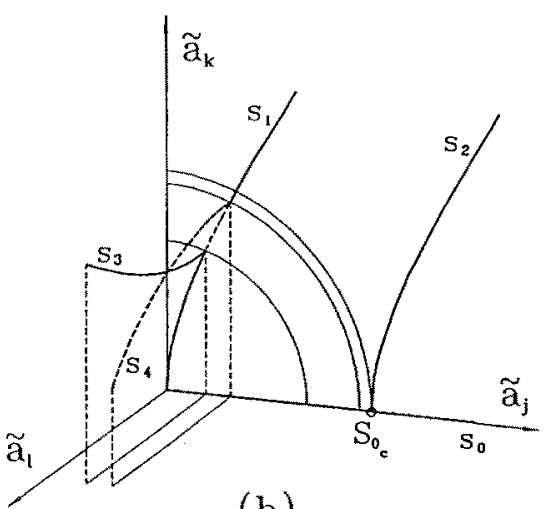

(b)

Fig. 2. Amplitude equilibrium paths for in-plane $k$-th and $j$-th primary resonant modes and (a) primary or (b) secondary out-of-plane $l$-th resonant mode. (-) stable, (- - ) unstable.

that admits the first integral of motion

$$
a_{j}^{2}+\frac{b_{j}}{b_{k}} a_{k}^{2}+\frac{c}{d} a_{l}^{2}=I_{j}
$$

and iso-energy surfaces are the spheres in the space of the normalized amplitudes: $\tilde{a}_{j}=a_{j}$, $\tilde{a}_{k}=\left(b_{j} / b_{k}\right)^{1 / 2} a_{k}, \tilde{a}_{l}=(c / d)^{1 / 2} a_{l}$.

\section{Steady-State Amplitude Oscillations and Bifurcation Analysis}

The steady-state solutions are the fixed points of (18) or (23). These equations admit solutions with two or three non-vanishing components; among the former attention is focused on the planar oscillations described by $a_{k} \neq 0, a_{j} \neq 0$ and $a_{l}=0$. The vanishing of the right hand side terms of (18) or (23) for $a_{l}=0$ gives

$$
\begin{aligned}
& \gamma_{k}= \pm \pi / 2 \\
& \left(b_{j} a_{k}^{2}-2 b_{k} a_{j}^{2}\right) \sin \gamma_{k}+\sigma_{k} a_{j}=0,
\end{aligned}
$$

while $\gamma_{l}$ remains undetermined. The relation $a_{k}-a_{j}$ furnishes two curves represented in Figure 2 for two cases of the illustrative example: (a) the prevailing in-plane second mode $(k=2, j=4)$ with the first out-of-plane mode $(l=1)$ in primary resonance; (b) the prevailing in-plane first mode $(k=1, j=2)$ with the first out-of-plane mode $(l=1)$ in secondary resonance. Curves $s_{1}\left(\gamma_{k}=-\pi / 2\right)$ emanate from the origin and represent the planar oscillations with the prevailing $a_{k}$ component which tend to the linear solution for small amplitude. Curves $s_{2}\left(\gamma_{k}=\pi / 2\right)$ bifurcate from the trivial path $a_{k}=0$ at the critical value $a_{j_{c}}=\sigma_{k} / 2 b_{k}$. However, this result is only an approximate value since a discrete model of continuous system able to accurately describe oscillations with prevailing $a_{j}$ should retain the component with frequency close to $2 \omega_{j}$, as previously observed.

An initial study of these curves includes an analysis of bifurcation with respect to an outof-plane disturbance. Stationary solutions with $a_{l} \neq 0$ require $\cos \gamma_{l} \equiv 0$; the last equation of (18) or (23) with $a_{l} \rightarrow 0$ furnishes the bifurcation conditions for the primary or secondary resonant disturbance, respectively. 
For each curve $s_{1}$ and $s_{2}$ (equation 25), depending on the system parameters, up to two points of bifurcation can exist for $\sin \gamma_{l}= \pm 1$. The corresponding levels of energy are reported in Figure 2.

The closed-form expression of the equilibrium paths with three non-vanishing components are obtained by zeroing the right side of the equations (18) or (23). On these curves originating from the bifurcation points constant values $\gamma_{k}= \pm \pi / 2$ and $\gamma_{l}= \pm \pi / 2$ are found, as on the other equilibrium paths.

The main characteristics of these curves can be deduced from the Figure 2. Three-mode solutions always bifurcate from curve $s_{1}$; for the primary resonance (a) only one bifurcation point is found, since the other is well outside the amplitude range considered, and then one three-mode solution $s_{3}\left(\gamma_{l}=-\pi / 2\right)$ appears; for the secondary resonance (b) the two three-mode bifurcated paths $s_{3}\left(\gamma_{l}=-\pi / 2\right)$ and $s_{4}\left(\gamma_{l}=\pi / 2\right)$ are contained in the planes $a_{j}=$ const.

In this last case (Figure $2 \mathrm{~b}$ ) a saturation of the $j$-th component occurs and an increment of energy goes only in the $k$-th and $l$-th components. In the former case (Figure $2 a$ ) the $k$-th component rapidly tends to saturation after bifurcation. In both cases the saturated components are those with frequency twice that of the bifurcated component, similar to what revealed in forced two degree-of-freedom systems with quadratic nonlinearities $[8,15,18]$.

The stability analysis of the three-mode solutions can be straightforwardly performed by means of the variational equations by determining the eigenvalues of the Jacobian evaluated at points of the curve. The results of the analysis are shown in Figure 2 where the stable (unstable) branches are solid (broken) lines. The stability of the two-mode solutions cannot be analyzed in the same way because the amplitude equations cannot be written in normal form, due to $a_{l} \equiv 0$. Cartesian representation of complex amplitudes must be used, as in [20, 25]. This technique has not followed here since it is dealt with as a particular case of a more general procedure suited to analyzing stability of periodic solutions which will be given in Section 7.

Figure 2 shows that at the bifurcation points the crossing paths exchange their stability characteristics; it is remarkable that in the secondary resonance the equilibrium looses stability after the first bifurcation point and regains it after the second. This agrees with results obtained in [22] by means of Mathieu equation theory. Instead the paths $s_{2}$ are always stable.

\section{Periodically Amplitude Modulated Planar Oscillations}

The planar motion is governed by three of equations (18) or (23) in which $a_{l} \equiv 0$ has been imposed:

$$
\begin{aligned}
a_{k}^{\prime} & =b_{k} a_{k} a_{j} \cos \gamma_{k} \\
a_{j}^{\prime} & =-b_{j} a_{k}^{2} \cos \gamma_{k} \\
a_{k} a_{j} \gamma_{k}^{\prime} & =\left(b_{j} a_{k}^{3}-2 b_{k} a_{k} a_{j}^{2}\right) \sin \gamma_{k}+\sigma_{k} a_{k} a_{j} .
\end{aligned}
$$

Some of the problems described by these equations are encountered in literature, and different techniques have been used to represent the solutions. Very effective representations have been given in [8,26]; in particular, in [8], by introducing two first integrals of motion $I_{1}$ and $I_{2}$, (26) are reduced to one differential equation:

$$
\zeta^{2}=4 b_{k} I_{1}\left[F^{2}(\zeta)-G^{2}(\zeta)\right]
$$






Fig. 3. Functions $F(\zeta)$ and $G(\zeta)$.

where $\zeta=a_{k}^{2} b j /\left(b_{k} I_{1}\right)$, and $I_{1}$ coincides with the energy $E=a_{j}^{2}+\left(b_{j} / b_{k}\right) a_{k}^{2}$. The functions $F(\zeta)$ and $G(\zeta)$, the last depending on the initial energy, are drawn in Figure 3; the intersections $\zeta_{1}$ and $\zeta_{2}$ of the two curves, at which the right hand side term of (27) vanishes, are inversion points of motion.

For any initial conditions, $\zeta$ varies periodically and so do the state variables $a_{k}, a_{j}$ and $\gamma_{k}$, giving a motion with periodically modulated amplitudes and phases in which the energy is continuously exchanged between the two modes. For a particular choice of initial conditions $G$ is tangent to $F$, which describes a steady-state oscillation. When $G$ intersects $F$ at the origin the double root $\zeta=0$ represents a stationary oscillation with only $a_{j} \neq 0$.

Here a different representation of the motion is given in the space of the state variables. For a certain energy level $E$, since the cyclic variable $\gamma_{k}$ does not appear in the integral of motion, the trajectories, numerically obtained from (26), lie on a cylinder.

Two different levels of energy are illustrated in Figure 4 where $\gamma_{k}$ varies in $[-\pi, \pi]$. For a low level (Figure 4a) one stationary two mode solution $S_{1}$ at $\gamma_{k}=-\pi / 2$ and one mode solution $S_{0}\left(a_{k}=0, \gamma_{k}\right.$ arbitrary) occur, according to the paths in Figure 2. Both solutions are stable. Weak amplitude modulation occurs on each trajectory, while strong phase modulation occurs on most of them, except for the closed trajectories which fill a small region around $S_{1}$.

This picture has similarities with the previous noose representation of Figure 3. Since $E=$ const, trajectories are associated to parallel lines: $G_{0}$ corresponds to the $S_{0}$ solution which is clearly stable; $G_{1}$ corresponds to the $S_{1}$ solution which again is stable, while the others define periodic amplitude modulations. In particular $G^{*}$ defines the largest closed trajectory where there is a complete energy transfer in $a_{k}$ and delimits the region of closed trajectories on the right from that of the open trajectories on the left.

As said in Section 2, all the two-mode solutions, either constant or periodic amplitude, are always stable for a perturbation of these components.

For a higher value of energy (Figure 4b) a new stationary solution $S_{2}$ appears. Another region of closed trajectories is generated, enclosed by a separatrix joining the two singular points $P_{1}$ and $P_{2}$ which makes the oscillation $S_{0}$ become unstable. They are located at 


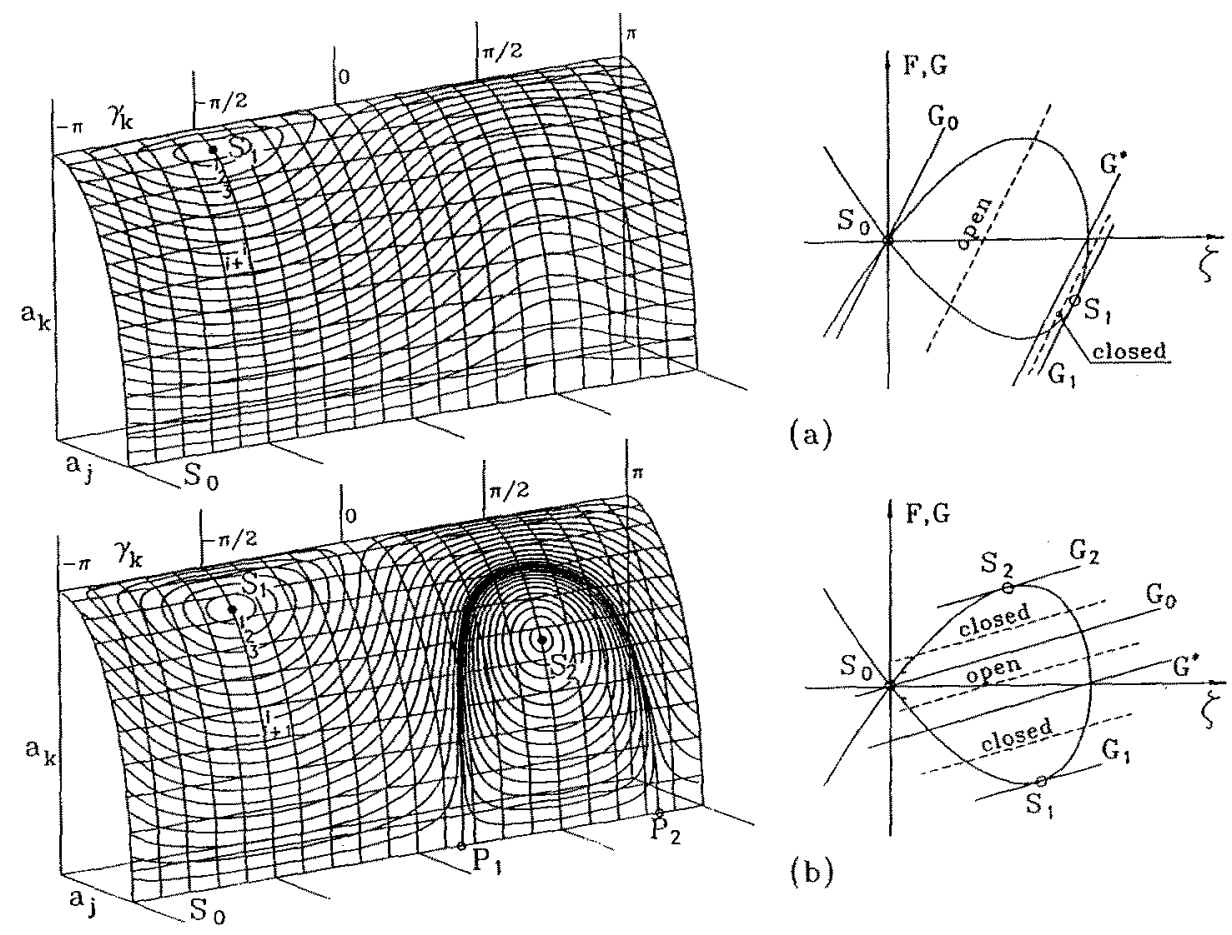

Fig. 4. Trajectories of nonstationary planar two-modes motions for (a) low and (b) high energy values. Comparison with the noose representation.

$\gamma=-\arcsin \left[\sigma_{k} /\left(2 b_{k} a_{j}\right)\right]$ obtained from the right hand side term of $\left(26_{3}\right)$ equated to zero for $a_{k}$ vanishing. In this case a strong modulation of amplitude and phase can occur. The phenomenon is confirmed on the noose representation where the slope of the line decreases as the energy increases and two tangent lines $G_{1}$ and $G_{2}$ can be found. The origin is now an unstable oscillation since a small perturbation of $G_{0}$ produces a periodic motion where the component $a_{k}$ increases irrespectively of the smallness of its initial perturbation.

Between the two situations described in Figure 4, lies the critical case in which the two singular points coincide and coalesce with $S_{2}$ for a critical value of energy $E_{o_{c}}=\left(\sigma_{k} / 2 b_{k}\right)^{2}$ at which the one component steady-state oscillation becomes unstable $\left(S_{o_{c}}\right.$ in Figure 2$)$. On the noose this critical condition occurs when $G_{0}$ is tangent to $F$ in the origin.

The knowledge of the $T$-periodic solution in the variables $a_{k}, a_{j}$ and $\gamma_{k}$ makes it possible to describe the time history of the displacements. According to (12 1$)$ and (16), $\theta_{k}$ and $\theta_{j}$ are evaluated from

$$
\begin{aligned}
& \theta_{k}^{\prime}=-b_{k} a_{j} \sin \gamma_{k} \\
& \theta_{j}^{\prime}=-b_{j} \frac{a_{k}^{2}}{a_{j}} \sin \gamma_{k} .
\end{aligned}
$$

They are the imaginary parts of (15) or (21) when $A_{l} \equiv 0$. Since the right hand side terms of (28) are $T$-periodic, denoting by $\nu_{n}(n=k, j)$ their mean value and absorbing the parameter $\varepsilon, \theta_{n}(t)$ can be written as

$$
\theta_{n}(t)=\nu_{n} t+\chi_{n}(t) \quad n=k, j,
$$





Fig. 5. Time histories of the total displacement and its components $v_{j}$ and $v_{k}$ at $l / 4$.

where $\chi_{n}(t)$ is a $T$-periodic function. By virtue of (29), a differentiation of $\left(17_{1}\right)$ leads to $\gamma_{k}^{\prime}=2 \nu_{k}-\nu_{j}+\sigma_{k}+2 \chi_{k}^{\prime}-\chi_{j}^{\prime}$. Since $\gamma_{k}$ is periodic, the mean value of its derivative is zero, i.e.,

$$
2 \nu_{k}-\nu_{j}+\sigma_{k}=0 .
$$

The displacement is thus

$$
v(s, t)=a_{k}(t) \varphi_{k}(s) \cos \left[\Omega_{k} t+\chi_{k}(t)\right]+a_{j}(t) \varphi_{j}(s) \cos \left[2 \Omega_{k} t+\chi_{j}(t)\right],
$$

where $\Omega_{k}=\omega_{k}+\nu_{k}$ is the nonlinear frequency and $\Omega_{j}=\omega_{j}+\nu_{j}=2 \Omega_{k}$ follows from (13) and (30).

The previous equations show clearly the meaning of the two terms of $\theta_{n}$ in (29): $\nu_{n}$ is the nonlinear modification of the mean instantaneous frequency and $\chi_{n}(t)$ accounts for the phase modulation. Moreover, nonlinearities adjust the frequencies of the two modes in the ratio 1:2 as in the stationary case. However, strictly speaking the displacement (31) describes an almost periodic oscillation because the period $T$ of the slow modulation is in general incommensurable with the period of the fast oscillation. In the steady oscillations $\chi_{n}(t)$ and $a_{n}(t)$ are constant.

In Figure 5, for the string oscillating in the prevailing first mode $(k=1)$ with the second companion one $(j=2)$, the amplitude modulated displacement (31) at $l / 4$ is plotted together with the two modal contributions $v_{k}(t)$ and $v_{j}(t)$. While each component shows an amplitude modulation associated with the internal resonance phenomenon, their combination produces a constant amplitude motion periodically shifted. This surprising result is due to the existence of a difference of phase between the two components.

The oscillation shape changes during a period due to the contributions of two modes oscillating with multiple frequencies. The picture of the deformed configuration at different instants in one period is shown in Figure 6, for two periods corresponding to zero or maximum drift, from which a travelling wave motion is appreciated. In particular, the case with zero drift (Figure 6a) also represents the behaviour of steady-state solutions. 


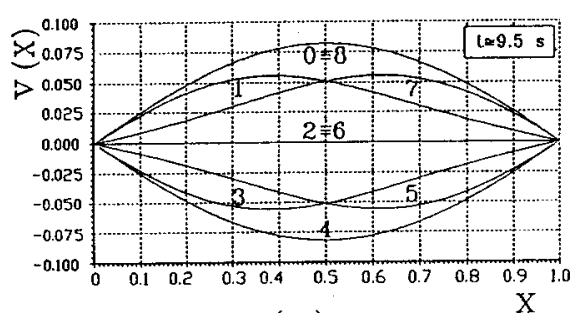

(a)

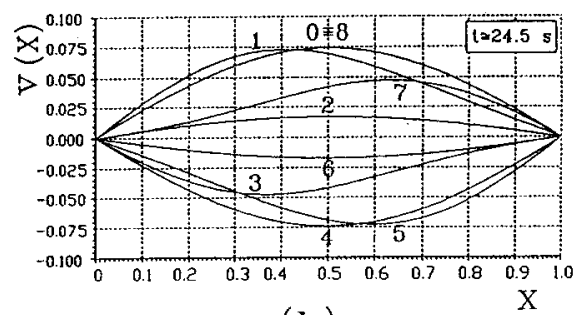

(b)

Fig. 6. Evolution of the deformed configuration in a period with (a) zero and (b) maximum drift around two different instants of the time histories in Figure 5.

\section{Stability Analysis of Periodically Modulated Oscillations}

\subsection{METHODOLOGY}

It is known that the stability analysis of planar solutions cannot be performed using (18) or (23) because they cannot be written in normal form when $a_{l} \equiv 0$. It is therefore necessary to go back to the amplitude equations and use the Cartesian form. However, these equations are non-autonomous; to make them autonomous the $A_{n}$ 's are transformed by the new complex amplitudes $B_{n}$ as follows:

$$
A_{n}=B_{n} e^{i \alpha_{n} t_{1}} \quad n=k, j, l,
$$

where $\alpha_{n}$ are real constants to be determined.

By referring to the primary resonance case, the use of (32) in (15) gives the autonomous system

$$
\begin{aligned}
& B_{k}^{\prime}-2 b_{k} \bar{B}_{k} B_{j}-2 c B_{l}^{2}+i \alpha_{k} B_{k}=0 \\
& B_{j}^{\prime}-2 b_{j} B_{k}^{2}+i \alpha_{j} B_{j}=0 \\
& B_{l}^{\prime}+2 d \bar{B}_{l} B_{k}+i \alpha_{l} B_{l}=0
\end{aligned}
$$

if $\alpha_{n}$ 's constants satisfy the conditions

$$
2 \alpha_{k}-\alpha_{j}+\sigma_{k}=0, \quad 2 \alpha_{l}-\alpha_{k}+\sigma_{l}=0 .
$$

Taking the variation of (33) around planar motion $B_{k_{0}} \neq 0, B_{j_{0}} \neq 0, B_{l_{0}} \equiv 0$ leads to

$$
\begin{aligned}
& \delta B_{k}^{\prime}-2 b_{k}\left(\bar{B}_{k_{0}} \delta B_{j}+B_{j_{0}} \delta \bar{B}_{k}\right)+i \alpha_{k} \delta B_{k}=0 \\
& \delta B_{j}^{\prime}-4 b_{j} B_{k_{0}} \delta B_{k}+i \alpha_{j} \delta B_{j}=0 \\
& \delta B_{l}^{\prime}-2 d B_{k_{0}} \delta \bar{B}_{l}+i \alpha_{l} \delta B_{l}=0 .
\end{aligned}
$$

If $\alpha_{k}=\nu_{k_{0}}$ and $\alpha_{j}=\nu_{j_{0}}$ are taken, with $\nu_{n_{0}}(n=k, j)$ frequency corrections of the planar periodic solution $a_{k_{0}}(t), a_{j_{0}}(t), \gamma_{k_{0}}(t),\left(34_{1}\right)$ is identically satisfied, as a consequence of (30), and (342) furnishes $\alpha_{l}$. Moreover, while the complex amplitudes $A_{n_{0}}(t)=$ $(1 / 2) a_{n_{0}}(t) \exp \left(i \theta_{n_{0}}(t)\right)$ are not periodic due to the form (29) of $\theta_{n_{0}}(t)$, the amplitudes $B_{n_{0}}(t)$ are periodic as follows from (32):

$$
B_{n_{0}}(t)=\frac{1}{2} a_{n_{0}}(t) e^{i \chi_{n_{0}}(t)} \quad n=k, j .
$$


Thus the introduction of $B_{n}$ variables made it possible to reconduct the stability analysis of the planar periodic motion to the solution of a standard variational problem with periodic coefficients.

In the simpler case of steady motions where $a_{n_{0}}$ and $\chi_{n_{0}}$ are constant, the procedure presented here is equivalent to that followed in $[20,25]$.

In equations (35), the out-of-plane component is uncoupled from the in-plane components. Since the two-mode periodic oscillations are always stable for in-plane disturbance, as easily argued from the description previously furnished, attention will be focused on the stability for an out-of-plane perturbation. This is fully described by $\left(35_{3}\right)$. By introducing the Cartesian form for $B_{n_{0}}$, the stability of the periodic planar motion is analyzed by means of the eigenvalues of the monodromy matrix determined by numerical integration of $\left(35_{3}\right)$.

In the particular case of steady oscillations, eigenvalues like $\lambda= \pm \sqrt{c}$, with $c$ real constant, are found; at the critical condition the two eigenvalues coalesce in the origin of the complex plane. In the periodic case the eigenvalues run along the unitary circle and coalesce in $\lambda=1$ at the critical condition.

For the sake of brevity the equations of the secondary resonance are not reported since they are obtained with the same procedure already shown; only numerical results are discussed below.

\subsection{RESULTS}

Some numerical results concerning the stability analysis of steady and periodic oscillations of the sample system under primary and secondary resonance are presented. The linearized analysis of stability illustrated above is used to characterize the motion and to determine the critical conditions. Subsequently the evolution of the state variables obtained by integrating the amplitude equations (18) or (23) are examined for some cases in the region of unstable motion.

The primary resonance case is dealt with first. It is useful to examine briefly the behaviour of steady solutions, whose stable and unstable branches have already been shown in Figure 2 . Unstable equilibrium points on the branch $s_{1}$ are perturbated by a small disturbance $a_{l}(0)$ and the evolution of the three amplitudes are drawn in Figure 7 . The steady motion is transformed into a periodic motion with a very regular exchange of energy from the planar modes to the out-of-plane mode. Along with higher values of energy, the transfer increases and its period become shorter. The trajectory $T_{1}$ in the $\left(\tilde{a}_{k}, \tilde{a}_{j}, \tilde{a}_{l}\right)$-space lies on an iso-energy surface, emanating from $S_{1}$ and passing through $S_{3}$, i.e. the three-mode steady-state solution on the bifurcated path (Figure $8 \mathrm{a}$ ). In the 5D state variables space the trajectory runs around $S_{3}$, what can be ascertained from projection shown in Figures $8 \mathrm{c}$ and $8 \mathrm{~d}$.

With regard to the periodically amplitude modulated oscillations it is found that, for an assigned level of energy, the $i$-th trajectory (Figure $4 \mathrm{a}$ ) cannot become unstable if the $(i-1)$-th trajectory is stable. Thus, for increasing levels of energy the instability of motion starts from the steady solutions $S_{1}$ and propagates toward external trajectories. This is highlighted in Figure $9 \mathrm{a}$ where the region of stable and unstable trajectories is defined on the section at $\gamma_{k}=-\pi / 2$ of the 3D state variables space. In accordance with the stability of $S_{2}$, all the closed trajectories around it are always stable.

The evolution of the amplitudes relevant to two iso-energy unstable orbits are represented in Figure 10. For the small orbits the evolution of their mean value is similar to that of the stationary solution (Figure 10a), while for the larger orbits the modulation of the mean value is 



Fig. 7. Amplitudes time histories for planar steady-state solutions $(k=2, j=4)$ with increasing energy perturbed by an out-of-plane primary resonant mode $(l=1)$. Label " 0 " denotes the critical case.

smoothed (Figure 10b) and disappears when the orbit falls into the stable region. Thus moving away from $S_{1}$, the faster modulation of amplitudes increases while the slower modulation of its mean value decreases.

The representation of one of the two orbits on the $\left(\tilde{a}_{k}, \tilde{a}_{j}, \tilde{a}_{l}\right)$ surface appears as in Figure $8 \mathrm{~b}$; the previously described phenomenon is well appreciated in Figures $8 \mathrm{c}$ and $8 \mathrm{~d}$ where it is apparent that the motion is quasi-periodic resulting in an orbit continuously moving up and down.

Similar qualitative behaviour is recognized in the secondary resonant motion, where, however, the most interesting aspect is the occurrence of the two close bifurcation points on the stationary branch $s_{1}$ which makes the steady oscillations lose and regain stability.

In Figure $9 \mathrm{~b}$ the stability regions on a section of state space are reported. It can be appreciated that for low levels of energy all oscillations are stable up to the first bifurcation; between the first and second bifurcation, the steady solutions and a certain region of close orbits are unstable while the furthest are stable; above the last bifurcation the steady solution and an increasing region of periodic orbits regain stability. In Figure 11, for the two levels of energy $E_{1}$ and $E_{2}$ indicated in Figure $9 \mathrm{~b}$, stable and unstable trajectories are reported on the cylinder. 



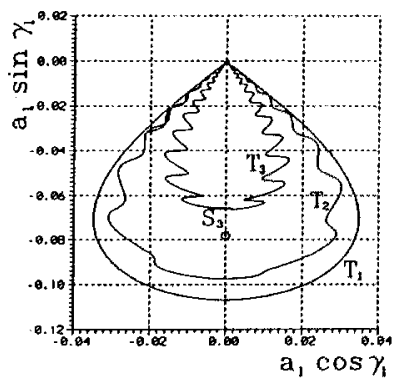

(c)

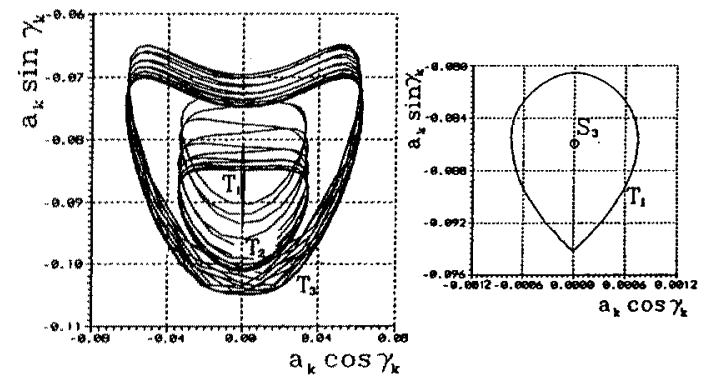

(d)

Fig. 8. Trajectories of the motion leaving the unstable steady-state solutions $\left(T_{1}\right)$ and the unstable periodic solutions $\left(T_{2}, T_{3}\right)$.

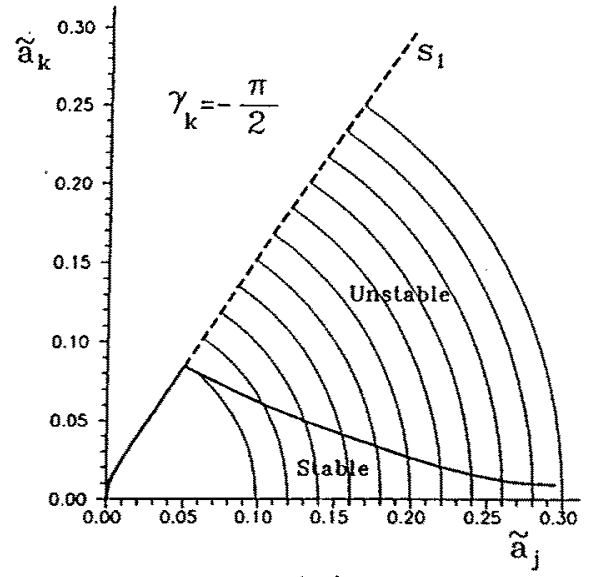

(a)



(b)

Fig. 9. Instability regions of the trajectories on the section at $\gamma_{k}=-\pi / 2$ in the $\left(a_{k}, a_{j}, \gamma_{k}\right)$-space: (a) primary and (b) secondary resonance.

\section{Conclusion}

The nonlinear free oscillations of an orbiting string satellite system are studied. The system is characterized by weak quadratic nonlinearities and presents some interesting features since it virtually works in conditions of simultaneous internal resonance. In principle a high number of modes could be involved in the motion, however, a two-mode model can give an approximate description of the oscillations with a prevailing $k$-th mode and a companion $j$-th mode in 

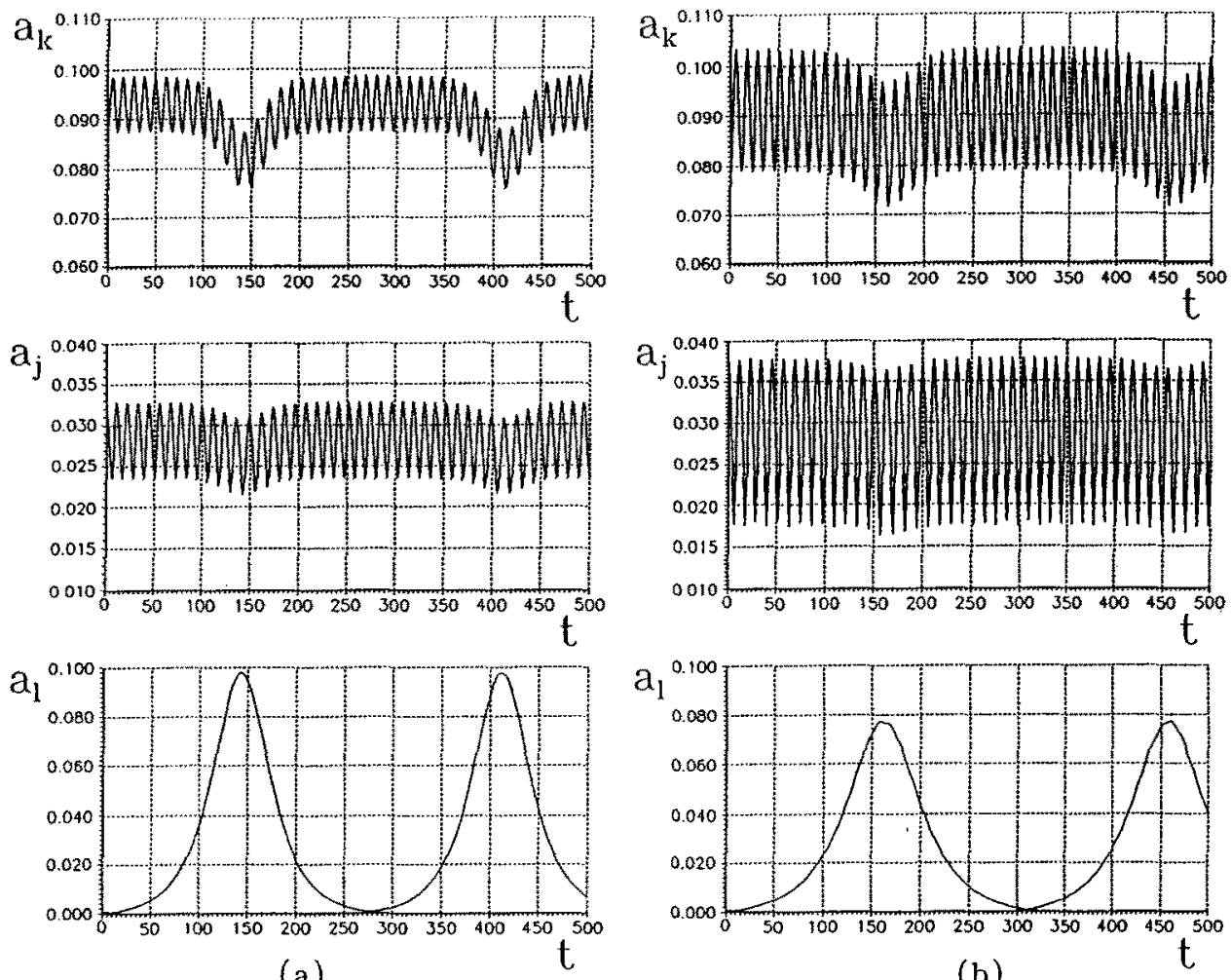

(a)



(b)

Fig. 10. Amplitudes time histories: unstable perturbed (a) small orbit and (b) large orbit.
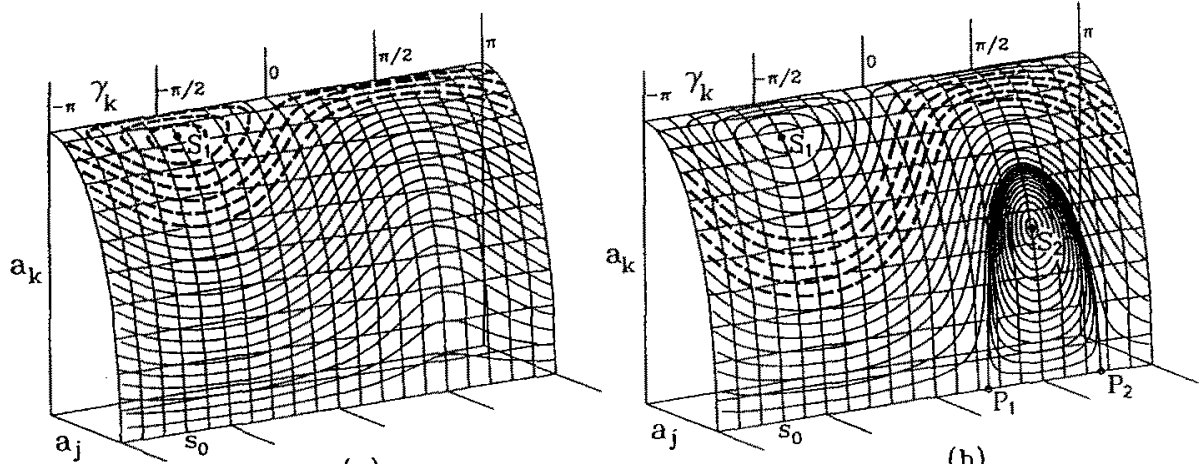

(a)

(b)

Fig. 11. Stable (-) and (- -) unstable planar periodic amplitude oscillations for the two levels of energy $E_{1}$ (a) and $E_{2}(\mathrm{~b})$ marked in Figure 9.

primary 1:2 resonance. Richer models are needed for the study of more complex motions. In particular a three-mode model is used to analyze bifurcations, stability and the evolution of unstable motions of planar oscillations with an out-of-plane component in primary or secondary resonance, that is a simultaneous internal resonance $2: 4: 1$ and $1: 2: 1$.

The stability of the steady and periodic amplitude oscillations is studied. The steady case when the disturbance involves a new component not present in the base oscillation has already been solved; it is shown here that a suitable transformation of the complex amplitudes can be introduced to bring the stability of periodic solutions back to the analysis of variational 
equations with periodic coefficients. Numerical results demonstrate that for a certain level of energy constant amplitude oscillations become unstable always before the periodic ones. At a level of energy for which steady oscillations are unstable, small orbits close to steady solutions are also unstable but there exist large orbits with strong phase and amplitude modulations which are stable. Planar two-mode resonant motion $\left(\omega_{j} \cong 2 \omega_{k}\right)$ perturbed by an out-of-plane mode $l$-th in primary resonance $\left(\omega_{k} \cong 2 \omega_{l}\right)$ loses stability at a certain value of oscillation amplitude, while when perturbed in secondary resonance $\left(\omega_{k} \cong \omega_{l}\right)$ the motion first loses but then regains stability, thanks to the existence of two close bifurcations points on the equilibrium path.

The numerical solution of the five-dimensional system of the amplitude equations furnishes a description of the unstable three-mode motions in condition of simultaneous internal resonances. Steady two-mode planar solutions bifurcate in periodic non-planar oscillations where the amplitudes of the involved three modes are slowly modulated with an energy transfer among in plane and out-of-plane modes. When unstable planar periodic solutions are perturbed out-of-plane, the amplitude modulations of the planar components remain practically unchanged but they occur around a mean value which is modulated on a slower scale, similar to the behavior of unstable steady motions.

\section{Acknowledgements}

This work was partially supported by Ministry of University and Scientific \& Technological Research (MURST-40\%, 1993).

\section{Appendix A}

The inner product in equations (7) is defined by

$$
H() \cdot u=\int_{D} H_{D} u \mathrm{~d} D+\int_{\Gamma} H_{\Gamma} u \mathrm{~d} \Gamma,
$$

were $H_{D}$ is the formal part of the operator in domain $D$ and $H_{\Gamma}$ is its representation on the boundary $\Gamma$. Accordingly:

$$
\begin{aligned}
M(\ddot{v}) \cdot \delta v= & \int_{0}^{1} \ddot{v} \delta v \mathrm{~d} s+\frac{1}{\gamma} \ddot{v}_{S} \delta v_{S} \\
L_{v}(v) \cdot \delta v= & \int_{0}^{1}\left(f v^{\prime} \delta v^{\prime}-3 \xi x_{0} \delta v\right) \mathrm{d} s-\frac{3}{\gamma} \xi x_{0}(1) \delta v_{S} \\
L_{w}(w) \cdot \delta w= & \int_{0}^{1}\left[f w^{\prime} \delta w^{\prime}+\left(1-3 \xi x_{0}\right) w \delta w\right] \mathrm{d} s+\frac{1}{\gamma}\left(1-3 \xi x_{0}(1)\right) w_{S} \delta w_{S} \\
B(v, \dot{v}) \cdot \delta v= & -2\left[\int_{0}^{1} \delta v \int_{0}^{s} v^{\prime} \dot{v}^{\prime} \mathrm{d} s \mathrm{~d} s+\int_{0}^{1} \delta v^{\prime} v^{\prime} \int_{1}^{s} \dot{v} \mathrm{~d} s \mathrm{~d} s\right] \\
& -\frac{2}{\gamma}\left[\delta v_{S} \int_{0}^{1} v^{\prime} \dot{v}^{\prime} \mathrm{d} s-\dot{v}^{\prime} \int_{0}^{1} \delta v^{\prime} v^{\prime} \mathrm{d} s\right]
\end{aligned}
$$


Table 1. Linear frequencies of transversal modes.

\begin{tabular}{rrrrrrr}
\hline & & $k=0$ & $k=1$ & $k=2$ & $k=3$ & $k=4$ \\
\hline \multirow{4}{*}{$l=5 \mathrm{~km}$} & $\omega_{v_{k}}$ & 1.736 & 23.022 & 45.845 & 68.712 & 91.590 \\
& $\omega_{w_{k}}$ & 2.004 & 23.044 & 45.856 & 68.719 & 91.595 \\
& $p_{k}$ & 0.238 & 3.160 & 6.292 & 9.431 & 12.571 \\
& & & & & & \\
& $\omega_{v_{k}}$ & 1.725 & 6.577 & 12.265 & 18.122 & 24.030 \\
& $\omega_{w_{k}}$ & 1.995 & 6.652 & 12.306 & 18.149 & 24.051 \\
& $p_{k}$ & 0.909 & 3.464 & 6.461 & 9.545 & 12.658 \\
\hline
\end{tabular}

$$
\begin{aligned}
& C(w, \dot{w}) \cdot \delta v=-2 \int_{0}^{1} \delta v \int_{0}^{s} w^{\prime} \dot{w}^{\prime} \mathrm{d} s \mathrm{~d} s-\frac{2}{\gamma} \delta v_{S} \int_{0}^{1} w^{\prime} \dot{w}^{\prime} \mathrm{d} s \\
& D(w, \dot{v}) \cdot \delta w=-2 \int_{0}^{1} \delta w^{\prime} w^{\prime} \int_{0}^{s} \dot{v} \mathrm{~d} s \mathrm{~d} s+\frac{2}{\gamma} \dot{v}_{S} \int_{0}^{1} \delta w^{\prime} w^{\prime} \mathrm{d} s .
\end{aligned}
$$

\section{Appendix B}

The following values of the system parameters are assumed for the illustrative example:

$$
\begin{array}{ll}
n^{2}=1.35 \times 10^{-6} \mathrm{~s}^{-2} & \mu=5.76 \times 10^{-3} \mathrm{~kg} / \mathrm{m} \\
a=\left(\mu_{e} / n^{2}\right)^{1 / 3}=6657 \mathrm{~km} & A=4 \times 10^{-6} \mathrm{~m}^{2} \\
m=500 \mathrm{~kg} . & E=7 \times 10^{10} \mathrm{~N} / \mathrm{m}^{2} \\
l=5 \mathrm{~km} . &
\end{array}
$$

The corresponding non-dimensionalized parameters (1) are:

$$
\alpha^{2}=6.94 \times 10^{-7}, \quad \gamma=\gamma=0.0576, \quad \xi=7.51 \times 10^{-4} .
$$

Frequencies of two systems with different string lengths and the other parameters equal to those of illustrative example are given in Table 1. Spatial frequencies $p_{k}$ of the associated modal shapes are also reported.

\section{Appendix C}

The coefficients of the equations (15) are as follows:

$$
\begin{aligned}
b_{k} & =\frac{1}{4 m_{k}}\left(b_{k j k}-\frac{\omega_{j}}{\omega_{k}} b_{k k j}\right), \quad b_{j}=\frac{1}{4 m_{j}} \frac{\omega_{k}}{\omega_{j}} b_{j k k} \\
c & =\frac{1}{4 m_{k}} \frac{\omega_{l}}{\omega_{k}} c_{k l l}, \quad d=\frac{1}{4 m_{l}} \frac{\omega_{k}}{\omega_{l}} d_{l l k} .
\end{aligned}
$$


Previous positions hold also for equations (21), except for the following

$$
c=-\frac{1}{4 m_{j}} \frac{\omega_{l}}{\omega_{j}} c_{j l l}, \quad d=\frac{1}{4 m_{l}} \frac{\omega_{j}}{\omega_{l}} d_{l l j},
$$

where

$$
\begin{aligned}
m_{k} & =M\left(h_{k}\right) \cdot h_{k} \quad \text { with } \quad h=\varphi, \psi \\
b_{i j k} & =B\left(\varphi_{j}, \varphi_{k}\right) \cdot \varphi_{i}, \quad c_{i j k}=C\left(\psi_{j}, \psi_{k}\right) \cdot \varphi_{i}, \quad d_{i j k}=D\left(\psi_{j}, \varphi_{k}\right) \cdot \psi_{i} .
\end{aligned}
$$

For the illustrative example the coefficients (39) assume the following values:

(a) primary resonance case $k=2, j=4, l=1$ :

$$
b_{k}=4.67, \quad b_{j}=1.17, \quad c=0.185, \quad d=0.731
$$

(b) secondary resonance case $k=1, j=2, l=1$ :

$$
b_{k}=2.40, \quad b_{j}=0.61, \quad c=0.185, \quad d=0.731 .
$$

\section{References}

1. Misra, A. D. and Modi, V. J., 'A survey on the dynamics and control of tethered satellite systems', in Proceedings of the NASA/AIAA/PSN International Conference on Tethers in Space, 1986, pp. 667-719.

2. Barnerjee, A. K. and Kane, T. R., 'Tether development dynamics', Journal of the Astronautical Science 30, $1982,347-365$.

3. Beletskii, V. V. and Levin, E. M., 'Dynamics of the orbital cable system', Acta Astronautica 12, 1985, 285-291.

4. von Flotow, A. H., 'Some approximations for the dynamics of tethered spacecraft', Journal of Guidance, Control, and Dynamics 11, 1988, 357-364.

5. Liangdong, L. and Bainum P. M. 'Effect of tether flexibility on the tethered shuttle subsatellite stability and control', Journal of Guidance, Control, and Dynamics 12, 1989, 866-873.

6. Pasca, M., Pignataro, M., and Luongo, A., 'Three-dimensional vibrations of tethered satellite systems', Journal of Guidance, Control, and Dynamics 14(2), 1990, 312-320.

7. de Matteis, G. and de Socio, L. M., 'Dynamics of a tethered satellite subjected to aerodynamics forces', Journal of Guidance, Control, and Dynamics 14, 1991, 1129-1135.

8. Nayfeh, A. H. and Mook, D. T., Nonlinear Oscillations, Wiley-Interscience, New York, 1979.

9. Szemplinska-Stupnicka, W., The Behaviour of Nonlinear Vibration Systems (Volume I \& II), Kluwer Academic Publishers, Dordrecht, The Netherlands, 1990.

10. Nayfeh, A. H. and Balachandran, B., 'Modal interactions in dynamical and structural systems', Applied Mechanics Reviews 42, 1989, 175-201.

11. Miles, J. W., 'Resonant nonplanar motion of a stretched strings', Acoustical Society of America Journal 75, $1984,1505-1510$.

12. Johnson, J. M. and Bajaj, A. K., 'Amplitude modulated and chaotic dynamics in resonant motion of strings', Journal of Sound and Vibration 128, 1989, 87-107.

13. O'Reilly, O. M. and Holmes, P., 'Nonlinear, nonplanar, and nonperiodic vibrations of string', Journal of Sound and Vibration 153, 1992, 413-435.

14. Ibrahim, R. A. and Barr, A. D. S., 'Autoparametric resonance in a structure containing a liquid, part II: Three mode interaction', Journal of Sound and Vibration 42, 1975, 181-200.

15. Nayfeh, A. H. and Mook, D. T., 'A saturation phenomenon in the forced response of systems with quadratic nonlinearities', in Proceedings of 8th International Conference on Nonlinear Oscillations, Prague, Czechoslovakia, 1978, pp. 511-516.

16. Sridhar, S., Mook, D. T., and Nayfeh, A. H., 'Nonlinear resonances in the forced responses of plates, part I: Symmetric responses of circular plates', Journal of Sound and Vibration 41, 1975, 359-373.

17. Bux, S. L. and Roberts, J. W., 'Nonlinear vibratory interactions in systems of coupled beams', Journal of Sound and Vibration 104, 1986, 497-520. 
18. Nayfeh, T. A., Asrar, W., and Nayfeh, A. H., 'Three-mode interactions in harmonically excited systems with quadratic nonlinearities', Nonlinear Dynamics 3, 1992, 385-410.

19. Sanders, J. A. and Verhulst, F., Averaging Methods in Nonlinear Dynamical Systems, Springer-Verlag, New York, 1985.

20. Lee, C. L. and Perkins, N. C., 'Three-dimensional oscillations of suspended cables involving simultaneous internal resonances', Proceedings of the ASME Winter Annual Meeting 144, 1992, 59-67.

21. Benedettini, F. and Alaggio, R., 'Motions and stability in three-dimensional oscillations of a suspended cable nonlinear model' (in Italian), in 7th Italian Conference on Computational Mechanics, Trieste, Italy, 1993, pp. 334-339.

22. Luongo, A. and Vestroni, F., 'Nonlinear free periodic oscillations of a tethered satellite system', Journal of Sound and Vibration 175, 1994, 299-315.

23. Pasca, A., Luongo, A., Pignataro, M., and Vestroni, F., 'Free dynamics of the shuttle-tethered-satellite system', Dept. of Structural and Geotechnical Engineering, Univ, of Rome, Italy, Rept. 3, 1987.

24. Nayfeh, A. H., Perturbation Methods, Wiley-Interscience, New York, 1973.

25. Nayfeh, A. H. and Raouf R. A., 'Nonlinear oscillation of circular cylindrical shells', International Journal of Solids and Structures 23, 1987, 1625-1638.

26. Struble, R. A. and Heinbockel, J. H., 'Resonant oscillations of a beam-pendulum system', Journal of Applied Mechanics 6, 1963, 181-188. 